CERN-ACC-2014-0332

Davide.Aguglia@cern.ch

\title{
Report
}

\section{Pulse Transformer Design for Magnet Powering in Particle Accelerators}

D. Aguglia

Keywords: accelerators, design, modelling, pulsed power converter, transformer

\begin{abstract}
This paper presents an optimal design procedure for pulse transformers fed by power converters for supplying pulsed current magnets in particle accelerators. Usually their volume must be minimized because of limited space available in the accelerator tunnel. Analytical and numerical models are presented as well as an experimental validation and a real case study where the design tool is exploited to derive a wide variety of solutions depending on the pulse transformers location with respect to the magnet position.
\end{abstract}

Presented at:

EPE

Lille, France

September, 2013 


\title{
Pulse Transformer Design for Magnet Powering in Particle Accelerators
}

\author{
Davide Aguglia \\ CERN-European Organization for Nuclear Research, \\ 1217 Geneva 23, Switzerland \\ Tel.: +41/(0)22 76755 49, E-mail: davide.aguglia@cern.ch
}

\section{Keywords}

«Accelerators», «Design», «Modelling», «Pulsed power converter», «Transformer».

\begin{abstract}
This paper presents an optimal design procedure for pulse transformers fed by power converters for supplying pulsed current magnets in particle accelerators. Usually their volume must be minimized because of limited space available in the accelerator tunnel. Analytical and numerical models are presented as well as an experimental validation and a real case study where the design tool is exploited to derive a wide variety of solutions depending on the pulse transformers location with respect to the magnet position.
\end{abstract}

\section{Nomenclature}

$\begin{array}{lllllr}B_{\max } & \text { Max. magnetic induction } & T & L_{m} & \text { Magnetising inductance } & m \\ d_{c} & \text { Central insulating distance } & m & L_{\sigma} & \text { Total leakage inductance } & H \\ d_{p c} & \text { Primary-core insulating distance } & m & L_{\sigma 1} & \text { Primary leakage inductance } & H \\ d_{p s} & \text { Primary-secondary insu. distance } & m & L_{\sigma 2} & \text { Secondary leakage inductance } & H \\ d_{w c} & \text { Windings-core insulating dist. } & m & n_{1} & \text { Primary turns number } & - \\ h_{t} & \text { Total transformer height } & m & n_{2} & \text { Secondary turns number } & - \\ h_{w} & \text { Windings height } & m & R_{1} & \text { Primary winding resistance } & \Omega \\ h_{y} & \text { Yoke height } & m & R_{2} & \text { Secondary winding resistance } & \Omega \\ I_{2, m a x} & \text { Secondary and max. sec. currents } & A & R_{m} & \text { Magnetising reluctance } A \cdot t u r n s / W b \\ J_{l}, J_{2} & \text { Prim. \& sec. current densities } & A / m^{2} & S_{c u l, 2} & \text { Tot. prim. \& sec, copper section } & \mathrm{m}^{2} \\ L_{c l} & \text { Primary cable length } & m & V_{2, m a x} & \text { Sec. and maximal sec. voltage } & V \\ L_{c 2} & \text { Secondary cable/strip-line length } & m & w_{t} & \text { Total transformer width } & m \\ L_{a v} & \text { Average core magnetic length } & m & \mu_{r} & \text { Iron relative permeability } & - \\ L_{i r o n} & \text { Iron length (3) } 3^{\text {dd }} \text { dimension) } & m & \varphi_{m} & \text { Magnetising magnetic flux } & W b \\ L_{t} & \text { Total transformer depth } & m & \rho_{c u} & \text { Copper resistivity } & \Omega \cdot m\end{array}$

\section{Introduction}

In particle accelerators magnets are often used to control the trajectory of the particles beam. Dipoles magnets are used to bend the beam in circular accelerators (synchrotrons) or to transfer it from an accelerator to the subsequent one or to an experimental area. Multi-pole magnets are also used to control the cross-sectional size of the particles beam (focusing and defocusing actions). In lower energy accelerators, magnets current is typically pulsed in order to reduce the energy consumption from the grid during dead times when there is no need for particle acceleration. Depending on the energy range of the accelerator, the current pulse length and amplitude vary. Typical values are pulse lengths in the $m s$-range and current amplitudes in the $k A$-range. A typical current shape requested to flow into such magnets is illustrated in Fig. 1. In the same figure (left) is presented the general layout for the powering system (usually with a four-quadrant converter). Notice that physically magnets are placed in tunnels, or dedicated galleries, where the accelerator lies. Usually the space inside the tunnel is quite limited, and the power converter is placed tens, or even hundreds, of meters away from the magnet, in an upper floor of the same building, or even in a different building. Carrying very high current in long cables or strip-lines implies high cost cables and power converter. 
a)

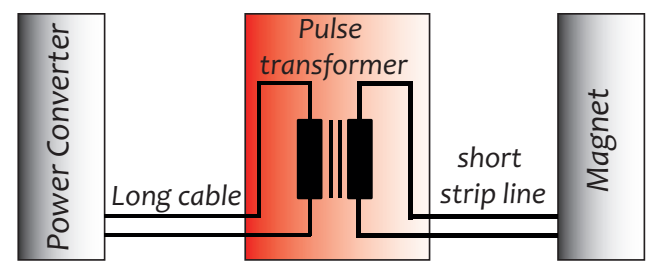

b)

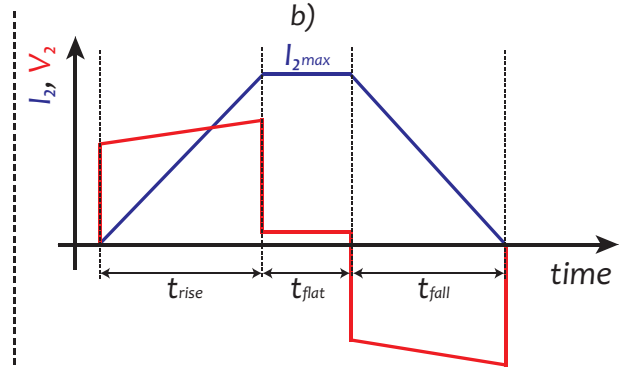

Fig. 1 - General magnet supply configuration (a); typical magnet current and voltage waveform (b).

For this reason pulse transformers are used to minimise cable cost and volume, and power converter cost. Pulse transformers must usually be installed inside the tunnel, imposing very challenging design optimisations for volume reduction. Considering the lack of literature on the design of such kind of pulsed power transformers [1], this paper presents a dedicated optimal design procedure. Furthermore, guidelines for the determination of a good specification for such kind of transformers are given to the power electronics engineer who requires integrating a pulse transformer coming from industry.

\section{Specifications for pulse transformer design}

It is of primary importance to correctly specify the pulse transformer in order to leave the designer maximum freedom for optimization while considering the minimal functional specifications. The specification analysis is based on the retained transformer equivalent circuit of Fig. 2(b), whereas Fig. 2 (c) is a photograph of a typical pulse transformer, used for validation purposes in this paper. The most important points to be treated in the specification of a dry-type pulse transformer (from the buyer point of view), are:

1. Provide secondary current and voltage shapes: From the specified magnet current shape, provide the RMS value and compute the secondary voltage shape considering $R_{m a g}$ and $L_{\text {mag }}$ of Fig. 2(b). From the secondary current harmonic content estimate the maximal $R_{\text {mag }}$ (skin effect) and consider it for an accurate and conservative secondary voltage shape.

2. Minimum magnetizing inductance $L_{m}$ : this value is related to the magnetizing current which must be provided by the power converter. The magnetizing inductance specification must be considered as a minimal value requirement, considering the maximal overcurrent allowed from the power converter for magnetizing the pulse transformer.

3. Transformer ratio: Specify the primary to secondary current ratio at nominal operating conditions (not the turn's ratio as the magnetizing inductance may widely influence the currents ratio).

4. Short-circuit current: the maximal allowable current in case of short-circuit at the transformer secondary connections should be provided. The designer needs this information in order to consider electro-dynamical efforts on windings. This may depend on protection systems of the power converter or on the maximal stored energy in it. If short primary cables are foreseen, one may have to specify the minimal transformer leakage inductance to limit the short-circuit peak current, however this practice must not impose too strong limitations to the transformer designer. If possible, do not impose leakage inductance values.

5. Surface temperature / efficiency: Specify the maximal transformer surface temperature at nominal operation and ambient temperature. Alternatively, if transformer volume is not a concern, the minimal efficiency can be specified.

6. Voltage insulation: Minimal insulation voltage of windings to ground and inter-windings, or standard class, must be specified along with the required test procedure to be performed before delivery.

7. Maximal overall dimensions. 
a)

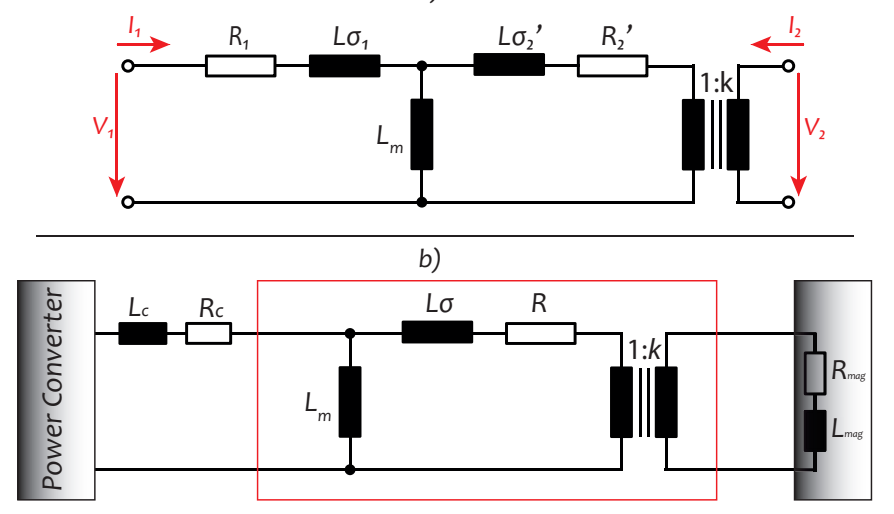

c)

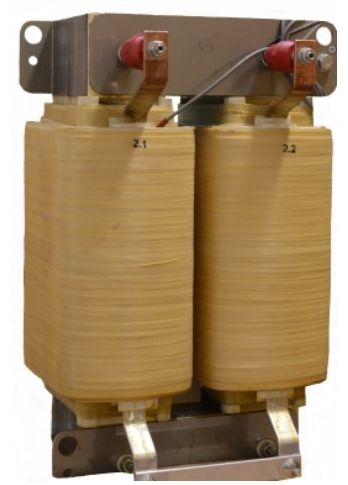

Fig. 2 - a) standard and b) simplified equivalent circuits of the pulse transformer; c) photograph of a typical $1.2 \mathrm{kA}$, pulse transformer with a structure of Fig. 4 (dimensions: $0.4 \mathrm{~m} \times 0.45 \mathrm{~m} \times 650 \mathrm{~m}$ ).

\section{Optimal Design procedure and models}

\section{Optimisation design procedure with automatic FEA correction}

The general transformer optimal design procedure is illustrated in Fig. 3 and includes a correction of the analytical model by Finite Element Analysis (FEA). This method was firstly presented in [2] and allows fast design optimization with a smart analytical model correction from FEA. It is demonstrated that for most designs the correction by FEA is used a few times only (less than 10), minimizing timeconsuming numerical computation. From the initial dimensions of a given transformer topology the analytical design model is used to derive the equivalent circuit parameters, allowing the analytical prediction of global performances. A non-linear optimization algorithm is used to find the best dimensions for a given performances objective, or criteria. When an optimal solution is found, the final geometry is automatically transferred to FEA for evaluation. FEA is used to numerically derive the equivalent circuit parameters, which are compared with the ones computed from the analytical design models. If there is disagreement, correction factors are computed to improve the analytical formulation. The optimization process re-starts based on the corrected analytical formulation. The process ends when there is agreement between the equivalent circuits parameters derived from the analytical and FEA formulation. This means that at the end of the process the solution is automatically validated by FEA.

The selected variables for the optimal design are $h_{w}, h_{y}, L_{i r o n}, S_{c u l}, S_{c u 2}$ and $B$ (refer to Nomenclature). The selected magnetic and insulating materials impose the following fixed parameter: $d_{p s}, d_{p c}, d_{w c}, \rho_{c u}$, $\mu_{r}$ and $B_{\max }$ (refer to Fig. 4 for geometrical definitions). The main optimisation constraints are: $\max J_{l}$, $\max J_{2}, B \leq B_{\max }$, $\min L_{m}$, and the main objective is the mass and volume minimisation. The choice of these variable and constraints is directly linked to the analytical modelling choices hereafter presented.

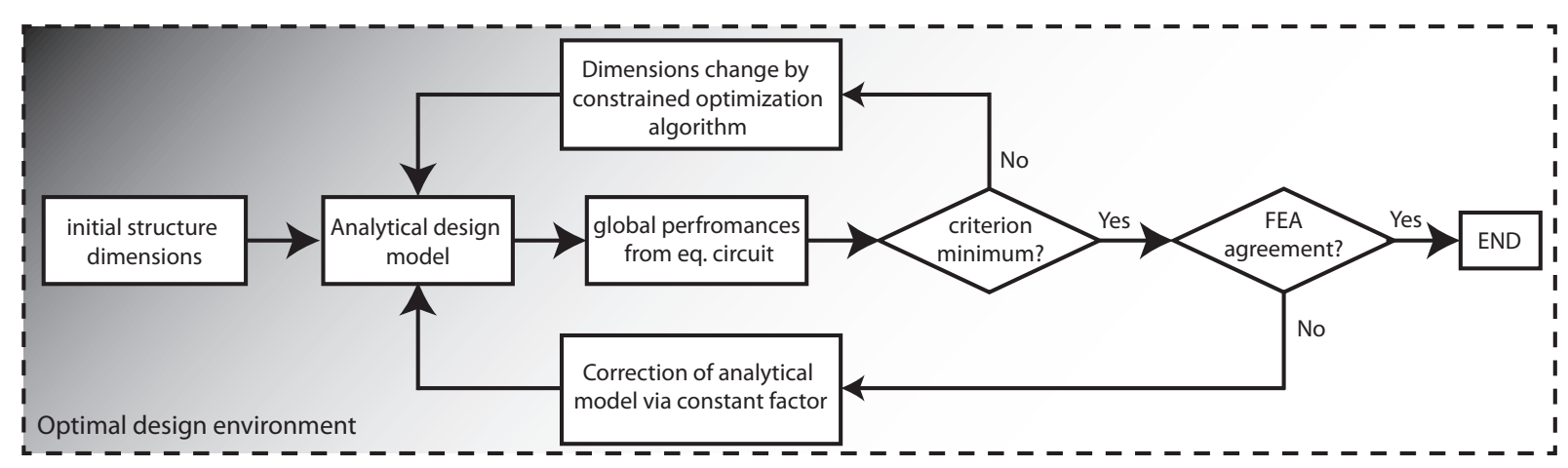

Fig. 3 - Pulse transformer design optimization procedure. 


\section{Analytical and numerical design models}

In this paper the most classical pulse transformer structure, depicted in Fig. 4 a), is analysed. This topology is the most used in the range of currents between $100 \mathrm{~A}$ and $5 \mathrm{kA}$, whereas the structure of Fig. $4 \mathrm{~b}$ ) is generally selected for higher currents. In Fig. 4 a) the primary winding is composed of 4 coils, 2 on each leg, which are enclosing the 2 coils of the secondary winding. This configuration allows minimising the total leakage inductance. All coils belonging either to the primary or secondary windings are connected in series. The main dimensioning equations are based on the assumption of no magnetic saturation. The voltage per turn can be calculated from (1) in case of current and voltage shape of Fig. 1. Furthermore it is assumed that the resistive voltage during $t_{\text {flat }}$ is negligible.

$$
v_{t}=\frac{d \varphi_{m}}{d t}=\frac{B \cdot S_{\text {iron }}}{t_{\text {rise }}}=\frac{B \cdot h_{y} \cdot L_{\text {iron }}}{t_{\text {rise }}}
$$

The number of turns of the primary winding can be obtained by $n_{1}=V_{1 \max } / v_{t}$, where $V_{\text {Imax }}$ is the maximal primary voltage, imposed by the power converter topology and switches technology. In order to compute the transformer performances the equivalent circuit parameters can be derived from the geometry dimensions. The magnetising inductance can be calculated from the magnetic reluctance (2).

$$
L_{m}=\frac{n_{1}^{2}}{R_{m}} \text { with } R_{m}=\frac{L_{a v}}{\mu_{0} \mu_{r} h_{y} L_{\text {iron }}}
$$

a)

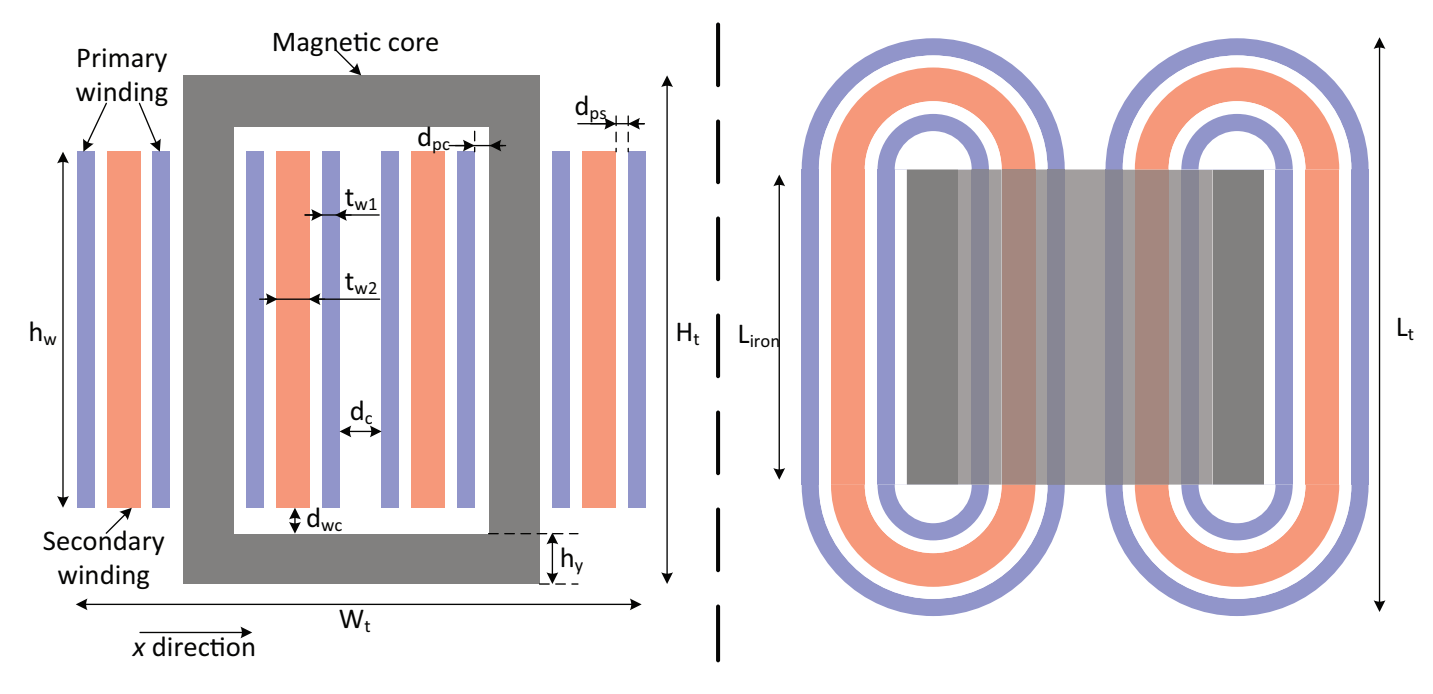

b)
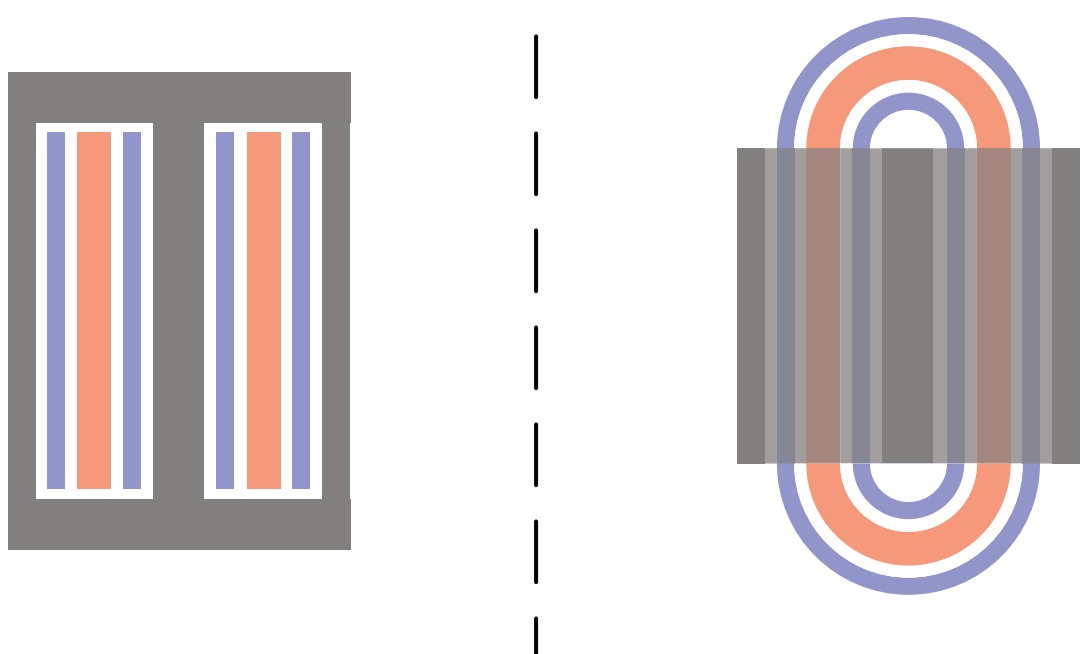

Fig. 4- Typical structure (cross-sectional views) and geometry definition of the pulse transformer. 
Leakage inductances can be analytically computed from the geometry in different ways [3]; image method, energy method, or flux element technique. Since the energy method will be used during the FEA calculation of these inductances, the same technique is adopted for the analytical calculation. With the assumption of an infinite magnetic permeability, and neglecting the end windings of the $3^{\text {rd }}$ dimension (right drawings of Fig. 4), the magnetic energy can be expressed as in (3).

$$
E_{m}=\frac{1}{2} \mu_{0} \iiint_{V} \vec{H}^{2} d V=\frac{1}{2} L_{\sigma} I_{1}^{2}
$$

Where $V$ denotes the volume of air and windings, and $L_{\sigma}$ the total leakage inductance referred to primary. Different regions of the volume $V$ should be analysed. To help identifying the different regions contributing to the leakage inductance, Fig. 5 shows the idealised magnetic field distribution along a cross-sectional view of the transformer, during a short-circuit operation, of a 2 coils windings transformer (left), and a 4 coils primary winding and 2 coils secondary winding transformer (right). The simplest regions for this analytical formulation are the volumes between primary and secondary windings. In fact, considering the hypothesis mentioned earlier, the magnetic field in these regions can be considered homogeneous and parallel to the height of the windings. The magnetic field in regions described by the distance $d_{p s}$ is:

$$
|\vec{H}|_{d p s}=\frac{n_{1} I_{1}}{n_{c 1}\left(h_{w}+2 d_{w c}\right)}
$$

Where $n_{c 1}$ is the number of primary coils in series, so that in (4) the number of turns per coil only is considered. In these regions $n_{l}$ and $I_{l}$ are constant, therefore, combining (3) and (4) the magnetic energies in these two regions can be easily derived as shown in (5).

$$
E_{m / d p s}=\frac{1}{2} \mu_{0} \iiint_{V}|\vec{H}|_{d p s}{ }^{2} d V=\frac{1}{2} \mu_{0}\left(n_{1} I_{1}\right)^{2} \frac{d_{p s} L_{\text {iron }}}{n_{c 1}^{2}\left(h_{w}+2 d_{w c}\right)}
$$

In the regions of the windings the magnitude of the magnetic field $H$ is linearly increasing along the $x$ axis (see Fig. 4 for $x$-axis definition). In the $t_{w l}$ regions, the magnetic field along the $x$-axis is varying as follows:

$$
\vec{H}(x)=\frac{n_{1} I_{1}}{n_{c 1}\left(h_{w}+2 d_{w c}\right)} \frac{x}{t_{w 1}}
$$

The magnetic energy inside one semi-coil of the primary winding is then expressed as in (7).

$$
E_{m / t w 1}=\frac{1}{2} \mu_{0} \int_{0}^{t w 1}\left(\frac{n_{1} I_{1}}{n_{c 1}\left(h_{w}+2 d_{w c}\right)} \frac{x}{t_{w 1}}\right)^{2}\left(h_{w}+2 d_{w c}\right) L_{\text {iron }} d x=\frac{1}{2} \mu_{0} \frac{\left(n_{1} I_{1}\right)^{2} L_{\text {iron }}}{n_{c 1}^{2}\left(h_{w}+2 d_{w c}\right)} \frac{t_{w 1}}{3}
$$

The total energy stored in the coils can be computed from the geometrical illustration of the magnetic field distribution of Fig. 5.
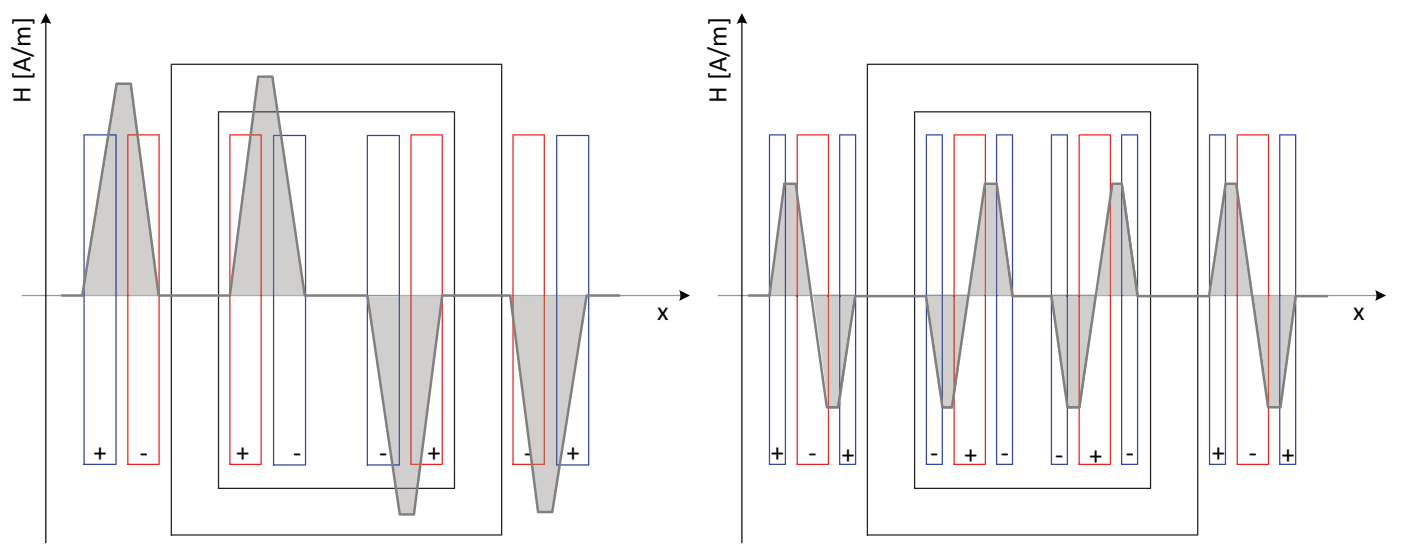

Fig. 5 - idealised magnetic field distribution along a cross-sectional view of a 2 coils primary windings transformer (left), and a 4 coils primary winding and 2 coils secondary winding transformer (right). 
For the windings configuration of Fig. 4 a) (or right side of Fig. 5), the energy in (7) must be multiplied by 8 for considering all primary coils, and the result must be multiplied by 2 in order to include the contribution of secondary coils. Combining (3), (6) and (7) one obtains the general expression of the total leakage inductance (8) for any winding configuration.

$$
L_{\sigma}=\frac{2 E_{m}}{I_{1}^{2}}=\mu_{0} n_{1}^{2} \frac{L_{i r o n}}{n_{c 1}^{2}\left(h_{w}+2 d_{w c}\right)}\left(k_{d p s} d_{p s}+k_{t w 1} \frac{t_{w 1}}{3}\right)
$$

For the structure depicted on the left of Fig. $5 ; k_{d p s}=4, k_{t w l}=4$ and $n_{c l}=2$, for the one on the right of Fig. $5 ; k_{d p s}=8$ and $k_{t w l}=8$ and $n_{c l}=4$.

The computation of the primary windings resistance is presented in (9), and the same method is applied for the secondary winding.

$$
R_{1}=k_{r} \frac{2 \rho_{c u} n_{1}^{2} A t l_{1}}{S_{c u 2}}
$$

$k_{r}$ is the skin effect coefficient and $A t l_{1}$ is the average turn length of the primary winding. For rectangular conductors the resistance correction factor $k_{r}$ can be derived from the Poynting vector formulation [4]. This coefficient can be expressed as in (10).

$$
k_{r}(f)=\frac{R_{A C}}{R_{D C}}=\xi \frac{\sinh (2 \xi)+\sin (2 \xi)}{\cosh (2 \xi)-\cos (2 \xi)}
$$

with:

$$
\xi=\alpha h \quad \& \quad \alpha=\sqrt{\frac{\pi \mu_{0} f}{\rho_{c u}}}
$$

where $\alpha$ is the inverse of the skin depth, and $h$ the height of the rectangular conductors. In such kind of transformers the secondary winding presents a very high conductor cross-section due to the high current. In high current transformers (typically $I_{2}>10 \mathrm{kA}$ ), the difficulty in bending, or winding, high cross-sectional copper conductors, and the loss in filling factor if Litz wire, leads to the choice of a multilayer thin copper or aluminium foil as a conductor. With the foil height corresponding to the coil height, and thickness selected depending on the required skin effect attenuation. In high current pulsed transformers, due to this mechanical problematic, secondary windings often present one or two turns. Copper losses are derived from the winding resistances (which consider skin effect) and currents. Iron losses are computed using the improved Generalized Steinmetz Equation (iGSE) [5] which considers non-sinusoidal magnetic flux [6]. For a further increase of precision predicting losses in the specific case of trapezoidal magnetic flux in the magnetic material (following the current shape of Fig. $1 \mathrm{~b}$ )), one may apply results from [7].

FEA is used to verify and correct the analytical models (Fig. 3). Magnetising and leakage inductances are computed in FEA for each intermediate optimal solution. The magnetising inductance is simply derived by supplying the primary winding and measuring the magnetic energy confined in the iron core volume. Then the well-known formula $E_{m}=\frac{1}{2} L_{m} I_{1}^{2}$ is used to derive $L_{m}$. Local magnetic saturation is neglected, however the global saturation effect is considered in average by selecting an appropriate constant $\mu_{r}$. It is also possible to consider saturation effects during the FEA, however, a better analytical design model based on reluctance networks should be selected for efficient correction purposes [8]. During this simulation one verifies that the maximal induction coincides with the imposed one during the optimisation process. Similarly, the total leakage inductance is computed by measuring the total magnetic energy in the air and windings, in a case where the primary and secondary currents correspond to a short-circuit operation, under the Kapp hypothesis of $n_{1} I_{1}-n_{1} I_{2}=0$. Both winding structures ( 2 or 4 coils for the primary windings) have been tested and analytical results were compared with FEA. 

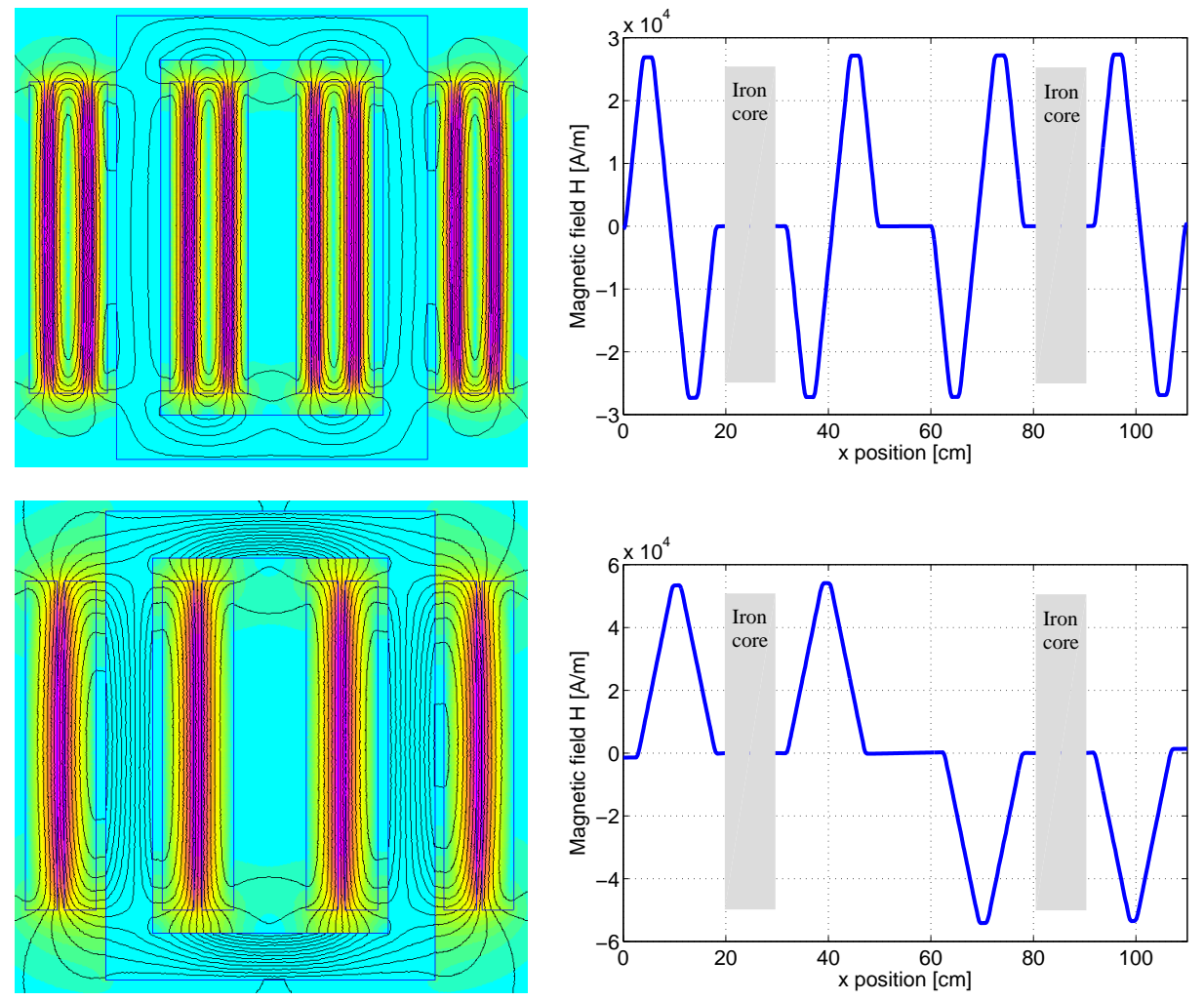

Fig. 6 - View of the 2D magnetic field $H$ distribution

Graphical results from FEA are depicted in Fig. 6, where for each winding structure (only the primary winding configuration changes) the magnetic field distribution at half of the height of the transformer has been measured (right side of Fig. 6) in order to verify the predicted $H$ field distribution of Fig. 5, used for the analytical formulation. In the case of arbitrary transformer dimensions (Fig. 6) maximal deviation of $15 \%$ between 2D FEA and 2D analytical formulation have been found on the leakage inductance, and $6 \%$ on the magnetising one.

\section{Design model experimental validation}

The design model has been validated on an existing $1.2 \mathrm{kA}$ pulse transformer, depicted in Fig. $2 \mathrm{c}$ ). The method consists in surveying all geometrical dimensions (magnetic core, coils, wires and insulating distances) of the existing transformer and using them into the analytical models which compute the equivalent circuit parameters. Afterwards all parameters are compared with the ones identified on the real transformer (Fig. 7). Table I shows all geometrical dimensions and the equivalent circuit parameters derived from experimental identification, analytical and numerical design models (the analytical model being not corrected in the procedure of Fig. 3). Experimental identification is based on [9]. Resistances are measured in DC prior to the non-linear identification method.

Table I - Transformer dimensions and parameters

\begin{tabular}{|c|c|c|c|c|c|c|c|}
\hline symbol & value & unit & parameter & Exp. & FEM & analytical & unit \\
\hline$\overline{\overline{h_{Y}}}$ & $\bar{~} 0.1$ & $\bar{m}$ & 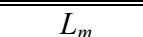 & 0.8 & 0.76 & 0.65 & 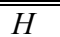 \\
\hline$d_{p c}$ & 0.001 & $m$ & $L_{\sigma}$ & 73 & 85 & 94 & $\mu H$ \\
\hline$d_{p s}$ & 0.001 & $m$ & $R_{l(D C)}$ & 51 & - & 56 & $m \Omega$ \\
\hline$d_{c}$ & 0.02 & $m$ & $R_{2(D C)}$ & 2.5 & - & 2.3 & $m \Omega$ \\
\hline$d_{w c}$ & 0.05 & $m$ & & & & & \\
\hline$t_{w 1}$ & 0.003 & $m$ & & & & & \\
\hline$t_{w 2}$ & 0.011 & $m$ & & & & & \\
\hline$h_{w}$ & 0.32 & $m$ & & & & & \\
\hline$L_{\text {iron }}$ & 0.172 & $m$ & & & & & \\
\hline
\end{tabular}

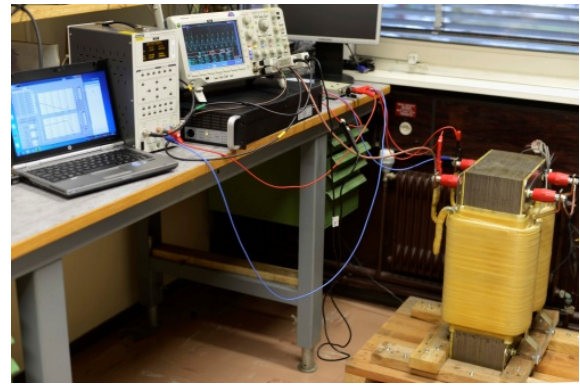

Fig. 7 - Transformer identification setup. 


\section{Optimal design example on a real case study}

Several optimisation designs have been carried out on a practical specification for a new set of magnets to be installed in the Proton Synchrotron Booster (PSB) at CERN. The specifications are $I_{2 \max }=6.8 \mathrm{kA}, t_{\text {rise }}=t_{\text {fall }}=5 \mathrm{~ms}$ and $t_{\text {flat }}=1 \mathrm{~ms}$, with a $2 \mathrm{~Hz}$ pulse repetition rate. The power converters can be placed at approximately $50 \mathrm{~m}$ of cable length from the magnets whose parameters are $L_{m a g}=13 \mu \mathrm{H}$ and $R_{m a g}=1.5 \mathrm{~m} \Omega$. Due to the limited space which could be allocated to the transformers in the accelerator tunnel (close to the magnets), the transformer design must minimise its volume. Furthermore, the dilemma arises regarding the location of the transformers: near the magnets (limited space), somewhere in a dedicated room between power converters and magnets, or inside the power converter rack. A wide number of transformer optimizations, with the method presented in Fig. 3, have been carried out to understand the global compromises against the distance between magnets and transformers (high current strip-lines, or cables, length of Fig. 1a)). A variable distance between transformers and magnets implies a variable cables inductance which, compared to the magnet one, may be very high for long cables. The considered inductance of the high current cables has been set to $0.5 \mu \mathrm{H} / \mathrm{m}$. Results are presented in Fig. 8, where three cases have been considered: 1. Maximal induction of $B_{\max }=1.5 T$ with $n_{2}=2$ (for a simplified mechanical construction of the transformer due to the high secondary conductor cross-section), 2. $B_{\max }=1.7 T$ with $n_{2}=2$, and $B_{\max }=1.7 T$ with no constraint on secondary turns number. For each case study 47 optimizations have been carried out (from a distance of $4 \mathrm{~m}$ to $50 \mathrm{~m}$ ). For the whole study the maximal primary voltage has been set to $800 \mathrm{~V}$ for allowing the use of standard $1.7 \mathrm{kV}$ IGBTs. Other optimization constants are presented in Table II, whereas the transformer structure is the one depicted in Fig. 4 a). The selected maximal current densities in Table II are valid for the peak current, the equivalent RMS current density being much lower. For each distance the total secondary inductance is different, leading to different maximal secondary voltages to impose the nominal magnet current. For higher distances, the peak secondary voltage must increase whereas the peak primary one is fixed at $800 \mathrm{~V}$. This leads, for all studied cases, to a decrease of transformer ratio with the increase of the distance as depicted in Fig. 8(a). Accordingly, the primary current increases with distance (Fig. 8(b)), and considering a constant primary $800 \mathrm{~V}$ voltage, the power converter apparent power rapidly increases with distance between power converters and magnets (Fig. 8(f)). The magnetising current (Fig. 8(c)) is increasing with distance as well, because of the necessary core flux increase to provide more secondary voltage with a fixed winding turns number $n_{2}$.

(a)

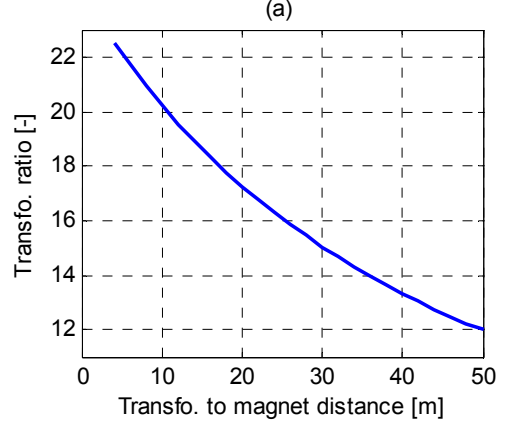

(d)

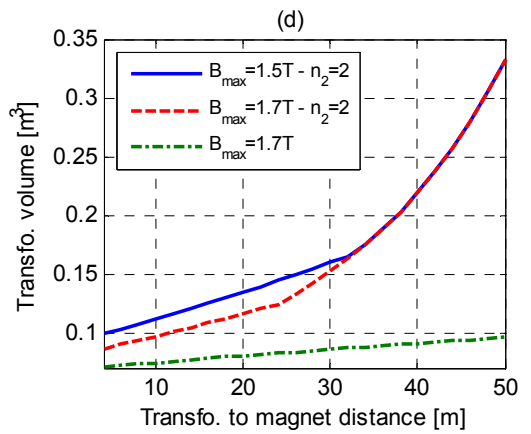

(b)

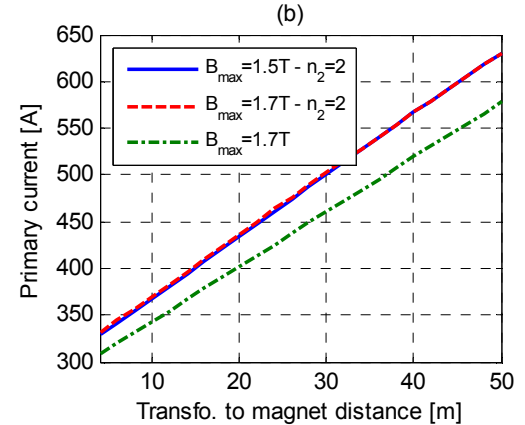

(e)

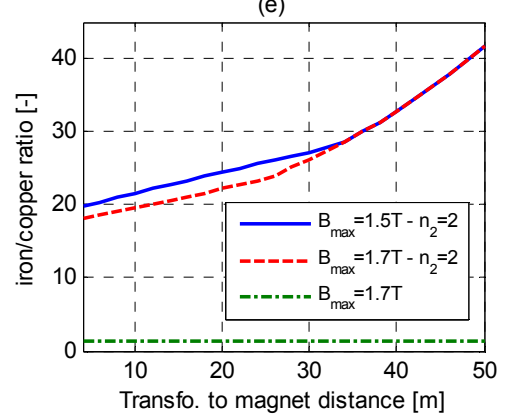

(c)

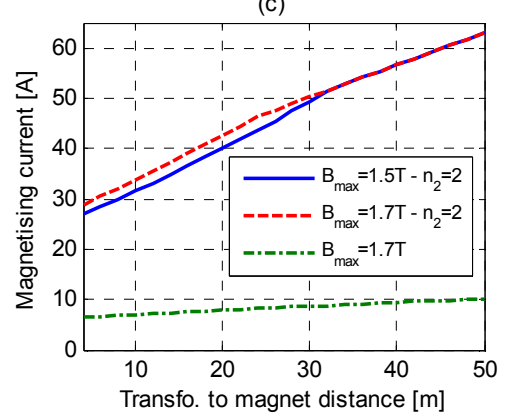

(f)

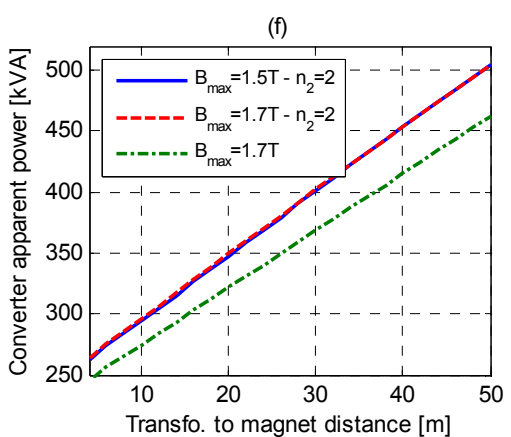

Fig. 8 - Transformer optimisation results for the three case studies. 
Table II - Optimisation constant and constrained parameters

\begin{tabular}{r|crl} 
unit & symbol & Value & Unit \\
\hline \hline Fixed maximum primary voltage (IGBT techn.) & $V_{I \max }$ & 800 & $\mathrm{~V}$ \\
Maximum magnetizing current (\% of $\left.I_{1}\right)$ & $I_{\operatorname{mag}}$ & 10 & $\%$ \\
Primary and secondary peak current densities & $J_{\text {Imax }}, J_{2 \max }$ & 8.3 & $\mathrm{MA} / \mathrm{m}^{2}$ \\
Copper filling factor & $k_{\text {fillcu }}$ & 0.7 & - \\
Homogeneous constant relative permeability & $\mu_{r}$ & 1000 & - \\
Primary to secondary distance & $d_{p s}$ & 5 & $\mathrm{~mm}$ \\
Core to primary distance & $d_{p c}$ & 10 & $\mathrm{~mm}$ \\
Central distance & $d_{c}$ & $20 \mathrm{~mm}$ \\
\hline \hline
\end{tabular}

In Fig. 8(d) notice how the transformer volume is increasing with distance. This phenomenon is also due to the fact that the secondary voltage per turn must be increased, leading to the necessity of increasing the iron core section for a given maximal induction. Therefore the iron to copper ratio of Fig. 8(e) increases with distance as well. For high distances with fixed $n_{2}=2$, the maximal magnetizing current limitation is active, leading to a further increase of core section (increase of $L_{m}$ ). In these regions the maximal magnetic induction must be decreased to keep the same magnetic flux excursion (same voltage), leading to a bad utilisation of the magnetic material. Much better results are obtained if the secondary number of turns is not fixed to $n_{2}=2$. However, this must be the subject of a trade-off analysis between transformer volume and cost variation due to difficult mechanical integration of a high current-high secondary turns winding (10 to 16 turns in this case). It is clear that the shorter the secondary cables the smaller the transformers and the power converter price; however, in this case civil engineering cost for placing the transformers near the magnet must be evaluated.

\section{Conclusion}

The presented optimal pulse transformers design method is efficient at quickly evaluating a wide variety of scenarios, where a global approach is essential. Nearly 150 pulse transformer optimizations with a 2D FEA correction method have been performed in less than 5 minutes on a standard personal computer to evaluate the global design compromises on a real case study. In the future it is foreseen to extend this methodology for integrating the power converter and the magnet design.

\section{References}

[1] Amoiralis E. I., Tsili M. A., Kladas A. G., Transformer Design and Optimization: A Literature Survey, IEEE Trans. Power Delivery, Vol. 24, No. 4, Oct. 2009, pp. $1999-2024$

[2] Cros J., Radaorozandry L. E., Figueroa J., Viarouge P., Influence of the magnetic model accuracy on the optimal design of a car alternator, International Journal for Computation and Mathematics in Electrical and Electronic Engineering (COMPEL), Vol. 27 No. 1, 2008, pp. $196-204$

[3] Margueron X., Besri A., Jeannin P.-O., Keradec J.-P., Parent G., Complete Analytical Calculation of Static Leakage Parameters : A Step Toward HF Transformer Optimization, IEEE Trans. Ind. Appl., Vol. 46, No. 3, May/June 2010, pp. $1055-1063$

[4] Chatelain J., "Machines Electriques - Volume X," Traité d'électricité series, Presses Polytechniques Romandes, Éditions Georgi, 1983, 630 p.

[5] Venkatachalam K., Sullivan C. R., Abdallah T., Tacca H., "Accurate prediction of ferrite core loss with nonsinusoidal waveforms using only Steinmetz parameters," in Proc. IEEE Workshop Comput. Power Electron., 2002, pp. $36-41$

[6] Aguglia, D., Neuhaus M., Laminated Magnetic Materials Losses Analysis under Non-Sinusoidal Flux Waveforms in Power Electronics Systems, EPE 2013, pp. $1-8$

[7] Muhlethaler J., Biela J., Kolar J. W., Ecklebe A., “ Improved core-loss calculation for magnetic components employed in power electronic systems, "IEEE Trans. Power Electr. Vol. 27, No. 2, 2012, pp. $964-973$

[8] Cros J., Taghizadeh M., Figueroa J.R., Radaorozandry L., Viarouge P., Simplified design model for fast analysis of large synchronous generators with magnetic saturation, IEEE Electrical Machines and Drives Conference (IEMDC), 2009, pp. $1515-1522$

[9] Aguglia D., Viarouge P., de Almeida Martins, C., Frequency-Domain Maximum Likelihood Estimation of High-Voltage Pulse Transformers Models Parameters, Accepted for IEEE Trans. Ind. Appl., Dec. 2013, pp. $1-10$ 